
Not for reproduction, distribution or commercial use.

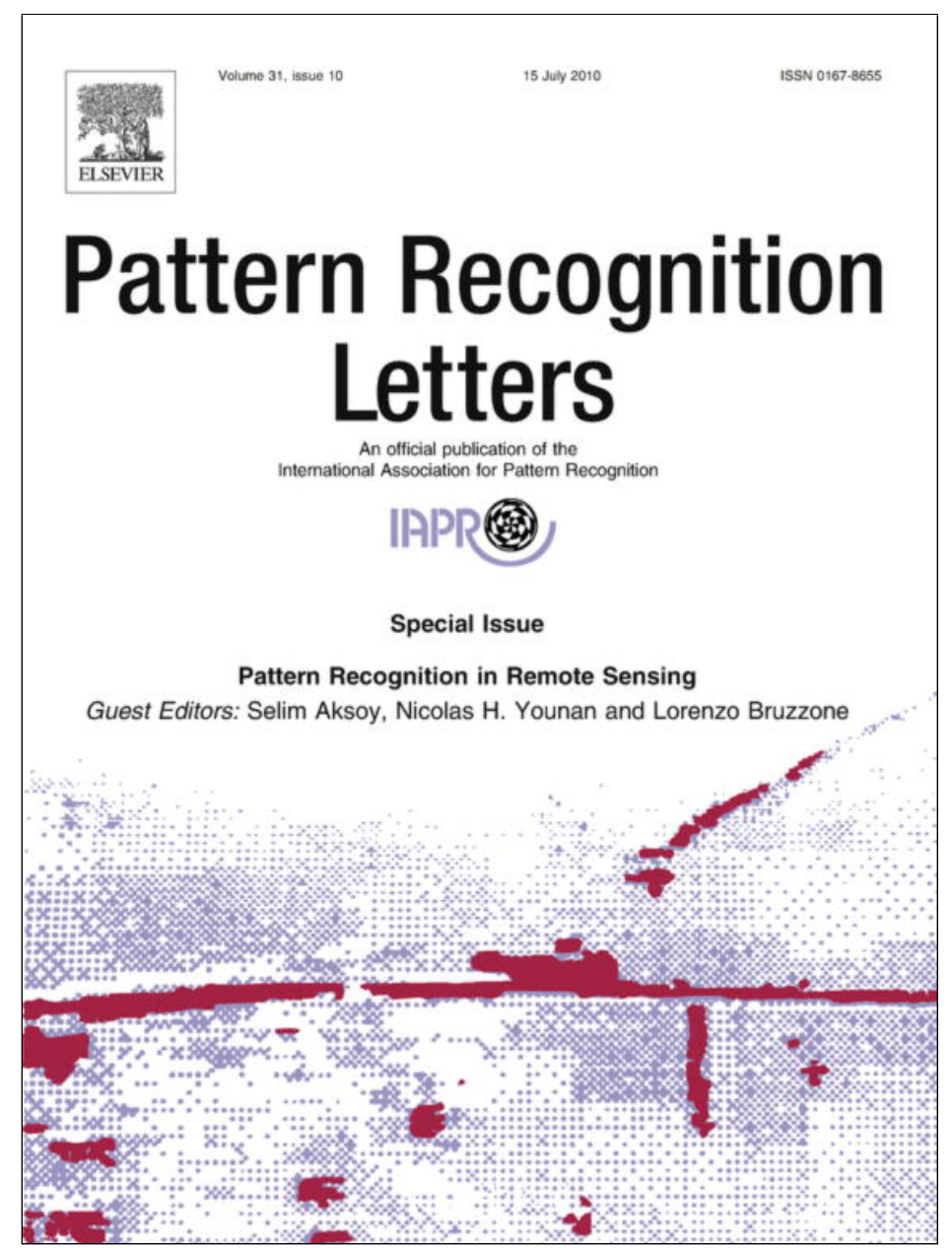

This article appeared in a journal published by Elsevier. The attached copy is furnished to the author for internal non-commercial research and education use, including for instruction at the authors institution and sharing with colleagues.

Other uses, including reproduction and distribution, or selling or licensing copies, or posting to personal, institutional or third party websites are prohibited.

In most cases authors are permitted to post their version of the article (e.g. in Word or Tex form) to their personal website or institutional repository. Authors requiring further information regarding Elsevier's archiving and manuscript policies are encouraged to visit:

http://www.elsevier.com/copyright 


\title{
Using local features to measure land development in urban regions
}

\author{
Beril Sırmaçek*, Cem Ünsalan \\ Computer Vision Research Laboratory, Department of Electrical and Electronics Engineering, Yeditepe University, Kayışdagı, İstanbul 34755, Turkey
}

\section{A R T I C L E I N F O}

\section{Article history:}

Available online 30 September 2009

\section{Keywords:}

Ikonos images

Gabor filtering

Local features

Spatial voting

Measuring land development

\begin{abstract}
A B S T R A C T
Monitoring urban development in a given region provides valuable information to researchers. Currently available, very high resolution satellite images can be used for this purpose. However, manually monitoring land development using these large and complex images is time consuming and prone to errors. To handle this problem, an automated system is needed to measure development in urban regions. Therefore, in this study we propose such an automated method to measure land development in a given urban region imaged in different times. We benefit from novel land development measures for this purpose. They are based on local features obtained from sequential images. As a novel contribution, we represent these local features in a spatial voting matrix. Then, we propose five different land development measures on the formed voting matrix. We test our method on 19 sets of sequential panchromatic Ikonos images. Our test results indicate the possible use of our method in measuring land development automatically.
\end{abstract}

(c) 2009 Elsevier B.V. All rights reserved.

\section{Introduction}

Land planning organizations, municipalities, disaster relief and environment protection agencies need to keep track of development in a prespecified region in time. Commercially available very high resolution satellite images (such as Ikonos or Quickbird) are suitable for this purpose. These grayscale images have at least $1 \mathrm{~m}$ spatial resolution and can be acquired in relatively short time intervals. They should be inspected periodically to detect urban region changes such as building construction and parkland formation. Unfortunately, this operation is time consuming and prone to errors if performed by a human expert. Therefore, researchers focused on developing automatic methods to detect changes and measure urban region development using satellite images.

There are fairly mature change detection algorithms in the literature such as Kosugi et al. (2004) and Mura et al. (2008). Radke et al. (2005) offers a detailed literature search on change detection methods. Although most change detection methods are powerful, they have two main deficiencies. First, these methods can not grade the change in time. Second, most of the change detection algorithms require perfect alignment between the two images to detect changes. To overcome the perfect alignment problem, $\mathrm{Li}$ et al. (2006) used Scale Invariant Feature Transform (SIFT) features to detect changes in urban objects. They first partitioned the image, then analyzed changes at a subgraph level. In a similar manner,

\footnotetext{
* Corresponding author. Tel.: +90 21657809 42; fax: +90 2165780400.

E-mail addresses: bsirmacek@yeditepe.edu.tr (B. Sırmaçek), unsalan@yeditepe. edu.tr (C. Ünsalan).
}

Tang and Prinet (2007) proposed a framework for structural change detection analysis using remotely sensed image pairs. They analyzed similarities and differences by calculating the distance of local features between the two images. The more advanced setting is not only detecting changes but also measuring them. Ünsalan (2007) introduced graph theoretical features to measure land development in grayscale satellite images. Although this method is very promising, it is computationally costly. Ünsalan (2009) extended his land development measures by introducing conditional statistical features defined on multispectral satellite images. If multispectral images are available, this method is fairly fast and powerful.

To extend our land development measures, we benefit from local features in this study. They are extensively used for object recognition in pattern recognition community. Recently, they have been used for urban region and building detection in satellite images (Sırmaçek and Ünsalan, 2009). In this study, we benefit from Gabor filtering and local maxima detection to extract local features. Then, we represent them in a spatial voting matrix. Based on this representation, we define five novel land development measures on a sequential image set acquired from the same location. In all our land development measures, we do not need perfect alignment (or preregistration). In fact, this is the main strength of our method. Our method also does not depend on the image size in the sequence. Therefore, scales of the images can slightly change due to the satellite's looking angle. After introducing our method, we test our novel land development measures on a diverse panchromatic Ikonos satellite image set and discuss their performances. We believe that, using a well-known method in the 
pattern recognition community (local features) in an important remote sensing problem (measuring land development) is a good example for joining both disciplines.

\section{Local feature extraction and representation}

We benefit from local features to measure land development in sequential satellite images. In this section, we explain how to extract them using Gabor filtering. Then, we represent local features in a voting matrix to be used in measuring land development in the next section.

\subsection{Gabor filtering}

Gabor filters are extensively used in texture segmentation and object recognition problems (Kyrki et al., 2004). They exhibit desirable characteristics of spatial locality and orientation selectivity (Vetterli and Kovacevic, 1995). Mathematically, the two-dimensional Gabor filter can be defined as the product of a Gaussian and a complex exponential function as:

$F_{\varphi}(x, y)=\frac{1}{2 \pi \sigma_{g}^{2}} \exp \left(-\frac{u^{2}+v^{2}}{2 \sigma_{g}^{2}}\right) \exp (j 2 \pi f u)$

where $u=x \cos \varphi+y \sin \varphi$ and $v=-x \sin \varphi+y \cos \varphi . f$ is the frequency of the complex exponential signal, $\varphi$ is the direction of the Gabor filter, and $\sigma_{g}$ is the scale parameter.

Gabor filtering can emphasize edge-oriented urban characteristics in satellite images. In order to detect these characteristics, we should use the most adequate $\sigma_{g}, f$, and $\varphi$ values for the given satellite image. Therefore, we designed a set of Gabor filters (with $\sigma_{g}=1.5$, and $f=0.65$ ) in which filter impulse responses are very similar to building edges in Ikonos satellite images. To cover differently oriented building edges, we picked $\varphi=\{0, \pi / 6,2 \pi / 6$, $3 \pi / 6,4 \pi / 6,5 \pi / 6\}$ radians. Hence, we filter the same image with six Gabor filters oriented in different angles.

\subsection{Local feature extraction}

To extract local features from a test image $I(x, y)$ with size $N \times M$, we benefit from the real part of the Gabor filter response as:

$G_{\varphi}(x, y)=\mathfrak{R} e\left\{I(x, y) * F_{\varphi}(x, y)\right\}$

where $*$ stands for the two-dimensional convolution operation. $G_{\varphi}(x, y)$ is maximum for image regions having similar characteristics with the filter. Therefore, we first search for the local maxima in $G_{\varphi}(x, y)$ for $x=1, \ldots, N$ and $y=1, \ldots, M$. If any pixel $\left(x_{0}, y_{0}\right)$ in $G_{\varphi}(x, y)$ has the largest value among its neighbors; we call it as a lo- cal maximum. This is a candidate for being a local feature. Next, we check the amplitude of the filter response $G_{\varphi}\left(x_{0}, y_{0}\right)$. We call our candidate local maximum $\left(x_{0}, y_{0}\right)$ as a local feature if and only if $G_{\varphi}\left(x_{o}, y_{o}\right)>\alpha$. To handle different images, we obtain $\alpha$ using Otsu's method (Otsu, 1979) on $G_{\varphi}(x, y)$ in an adaptive manner for each image separately. Therefore, we also eliminate weak local maxima in future calculations.

To represent each local feature further, we assign a weight, $w_{o}$, to it as follows. We first threshold $G_{\varphi}(x, y)$ with $\alpha$ and obtain a binary image $B_{\varphi}(x, y)$. In this image, pixels having value one correspond to strong responses. We obtain connected pixels to $\left(x_{0}, y_{0}\right)$ in $B_{\varphi}\left(x_{o}, y_{o}\right)$. By definition, in a binary image two pixels are connected to each other if there is a path (of pixels with value one) connecting them (Sonka et al., 2007). As we obtain all the connected pixels to $\left(x_{0}, y_{0}\right)$, we assign their number as the weight $w_{0}$. We expect the candidate local features to represent human activity such as building clusters. Unfortunately, all local features may not represent reliable information on the human activity. Therefore, we discard candidate local features having weight, $w_{0}$, less than 20 pixels. Finally, we obtain local features for the given $\varphi$ direction.

We apply this procedure in all $\varphi$ directions and obtain a total of $\mathrm{K}$ local features as $\left(x_{k}, y_{k}\right)$ with their weights $w_{k}$ for $k=1, \ldots, K$. We expect these local features to be located on building edges in the image. We provide such an example on the sample Adana $_{1}$ test image in Fig. 1. As can be seen, most local feature points are located on the building edges in this test image.

\subsection{Local feature set representation}

As we obtain local features and their weights, we form a voting matrix to measure land development. Our rationale in forming the voting matrix is as follows. Since each local feature possibly represents a part of a building or a man-made structure, it possibly indicates the overall human activity in the given image.

We define a voting matrix based on extracted local features as follows. We assume that, around each local feature there is a high possibility of an urban characteristic (such as a building). Therefore, each local feature has the highest vote at its spatial coordinate $\left(x_{k}, y_{k}\right)$ and its votes decrease w.r.t. spatial distance. Based on this definition, for an $N \times M$ sized image, we form the voting matrix for $x=1, \ldots, N$ and $y=1, \ldots, M$ as:

$V(x, y)=\sum_{k=1}^{K} \frac{1}{2 \pi \sigma_{k}^{2}} \exp \left(-\frac{\left(x-x_{k}\right)^{2}+\left(y-y_{k}\right)^{2}}{2 \sigma_{k}^{2}}\right)$

where $\sigma_{k}$ is the parameter for voting proximity for each local feature. For Ikonos images, we pick $\sigma_{k}=5 \times w_{k}$ to add some tolerance for voting. Here, $w_{k}$ is the weight of the local feature. If $\sigma_{k}$ has higher
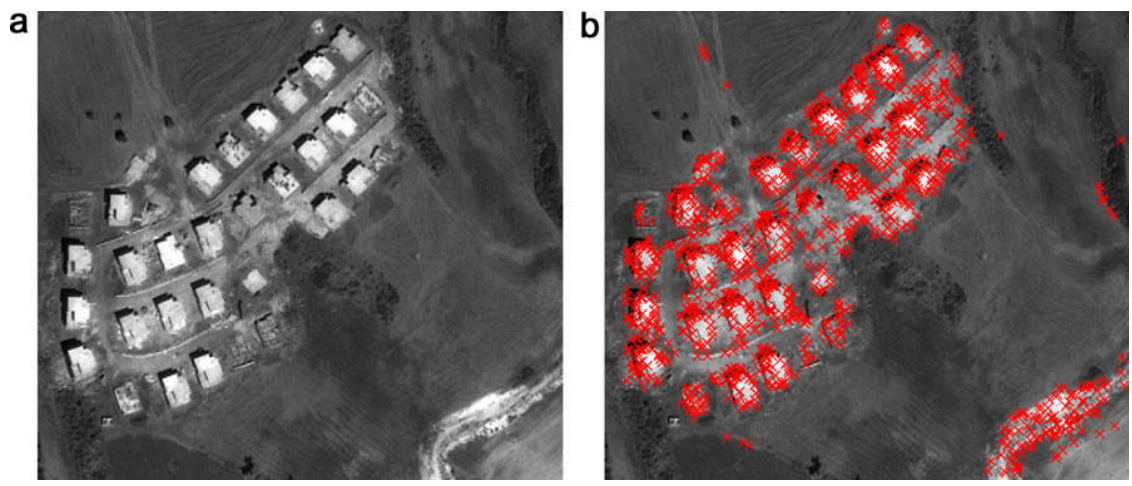

Fig. 1. The Adana $_{1}$ test image, local features extracted, as red crosses. (For interpretation of the references to colour in this figure legend, the reader is referred to the web version of this article.) 


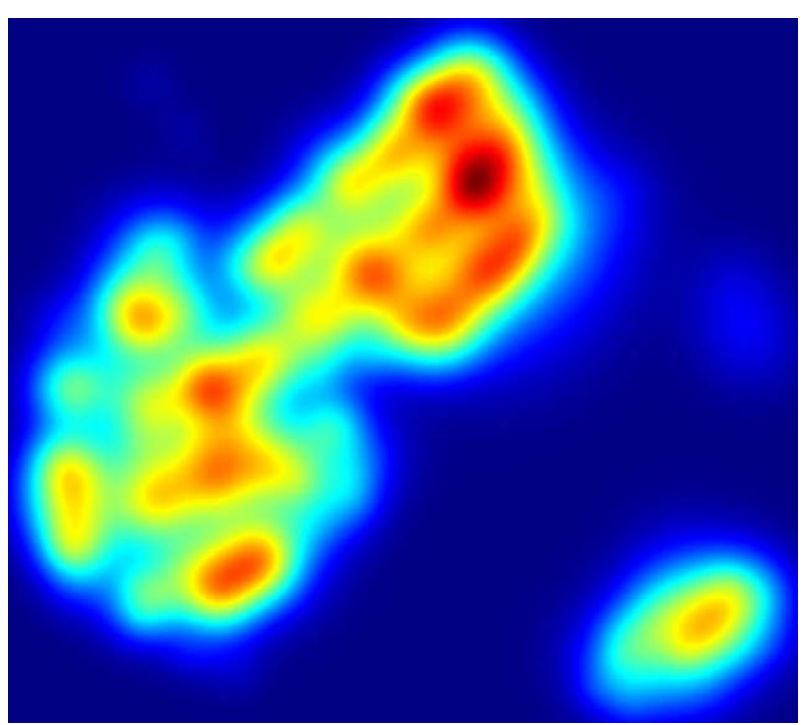

Fig. 2. The voting matrix in color coded form (red corresponds to the highest and blue to the lowest vote value) for the Adana $a_{1}$ test image. (For interpretation of the references to colour in this figure legend, the reader is referred to the web version of this article.)

values, each local feature will have a wider spatial effect in the voting matrix. On the other hand, if $\sigma_{k}$ has lower values, each local feature will have a narrower spatial effect.

For the Adana $_{1}$ test image, we provide the voting matrix in Fig. 2. In this figure, vote values are color coded (red corresponds to the highest and blue to the lowest vote value). As can be seen, votes are cumulated around buildings.

\section{Measuring land development}

After we obtain the voting matrix (possibly representing the human activity in a given satellite image), we introduce five novel land development measures on it. These measures are designed to summarize the voting matrix in various ways. We measure the development in a given urban region with its sequential images in time as follows. We calculate the specified land development measure for each image in the sequence. We expect these measures to indicate development either by increasing or decreasing w.r.t. time. Next, we introduce each novel land development measure.

\subsection{Number of voting local features}

Our first land development measure is based on the total number of voting local features in a given image. As mentioned above, we designed Gabor filters to detect possible man-made structures such as buildings. Local features are based on these filter responses. Therefore, in a given image if the number of man-made structures increase, we expect to have more local features. Hence, we define our first land development measure as the total number of local features as $m_{1}=K$.

\subsection{Normalized sum of votes}

As we mentioned above, the voting matrix is formed by the votes of local features in the given image. For more developed regions, we expect to have more votes in the voting matrix. The reason for this is twofold. First, we will have more local features in more developed regions. Second, we expect each local feature in a developed region to have more votes. Therefore, the sum of votes in the voting matrix may represent the development. We obtain our second land development measure on an $N \times M$ image as:
$m_{2}=\frac{1}{N M} \sum_{x=1}^{N} \sum_{y=1}^{M} V(x, y)$

We apply normalization by the image size, since all images in the sequence may not have the same size.

\subsection{Maximum vote}

Related to the normalized sum of votes measure, we can also benefit from the maximum vote from the voting matrix to measure land development. In the normalized sum of votes measure, we take all the votes in the voting matrix into account. However, this may be misleading for some development regions (such as the ones having occluded buildings). Therefore, we define the maximum vote value as our third land development measure as:

$m_{3}=\max _{(x, y)} V(x, y)$.

\subsection{Normalized urban area}

We also measure the land development by extracting the possible urban region in a given image. Our hypothesis is that, as the development increases in a region, the urban region also grows there. At this point, the voting matrix helps us to detect the possible urban region in a given satellite image. Remember, we constructed the voting matrix by the votes of local features possibly representing man-made structures. Therefore, if the voting matrix is thresholded in an appropriate way, the urban region may also be detected.

We first explain how to obtain the threshold value. Assume that, we have $T$ sequential images from a region. Therefore, we have $T$ voting matrices each separately corresponding to an image. As we mentioned in the previous sections, more votes possibly correspond to more developed regions. We have this information as our second measure, the normalized sum of votes, $m_{2}$. Since we have $T$ images in the sequence, we have $m_{2}^{t}$, for $t=1, \ldots, T$. We take the maximum $m_{2}^{t}$ as our benchmark and obtain the optimum threshold value for the corresponding voting matrix using Otsu's method (Otsu, 1979). Let's call this threshold value $a$. For each image having size $N \times M$ in the sequence, we calculate our fourth land development measure as:

$m_{4}=\frac{1}{N M} \sum_{x=1}^{N} \sum_{y=1}^{M}(V(x, y)>a)$.

\subsection{Normalized sum of votes in the urban region}

We finally measure the development using the normalized sum of votes in the urban region. In the previous section, we explored how to extract the possible urban region in the given image. We also based our fourth land development measure by the normalized urban area as in Eq. (6). Here, we extend this measure by also adding normalized sum of votes in the extracted urban region. Then, our fifth land development measure for an $N \times M$ image becomes:

$\left.m_{5}=\frac{1}{N M} \sum_{x=1}^{N} \sum_{y=1}^{M}(V(x, y)>a) \times V(x, y)\right)$

where the multiplication operation is on a pixel basis in Eq. (7). Using this formulation, we want to benefit from both the area and sum of votes in the urban region.

Besides these five novel land development measures, we also fuse them using our previous fusion method (Ünsalan, 2007). 
There, we normalized our measures and obtained their fusion by taking their mean values.

\section{Experimental results}

We test our five novel land development measures and their fusion on our data set introduced in (Ünsalan, 2007). In this data set, we have 19 different urban regions having diverse characteristics. Each urban region is imaged in either three or four different times. Therefore, we have a total of 69 images. We expect our land development measures to indicate the degree of development automatically on these image sets. To test our measures, we first provide two construction zones with their sequential images and corresponding measure values. Then, we apply our land development measures on 19 different urban regions. Finally, we compare the overall performance and computational cost of our novel land development measures with the existing ones in the literature.

\subsection{Sample results}

To explain our land development measures, we pick two representative urban regions $\left(\right.$ Adana $_{1}$ and Adana $_{4}$ ) as given in Fig. 3 . The first urban region, Adana $_{1}$, corresponds to a building construction zone. This region is a bare land in the first image. In the second and third images in the sequence, the buildings become visible. We expect our land development measures to automatically indicate this activity. The second urban region, Adana $_{4}$, corresponds to a parkland formation. In the first image, this region is also a bare land. In the second and the third images in the sequence, the development is visible. In Fig. 3, we also provide the fusion of land development measures for each test image sequence (in the first and second rows) separately.
As can be seen in Fig. 3, the fusion of land development measures correctly indicates the development in both image sequences by increasing its value. Therefore, we have a perfect performance on these two image sequences. On the Adana ${ }_{1}$ test image sequence, all other measures also performed perfect. However, for the $\mathrm{Adana}_{4}$ image sequence, measures $m_{1}$ and $m_{3}$ could not perform perfect. Both measures missed one development step and assigned it as less developed compared to the previous image in the sequence. The reason for this shortcoming is possibly the type of development activity there.

\subsection{Overall performance}

We also test our five land development measures and their fusion on 19 urban regions. We obtain the overall performance of our measures in accordance with our previous evaluation method given in (Ünsalan, 2007). There, to assess the performance of a feature, we define an ordinal position for each image in the sequence from one to three or four based on the advancement in construction. Next, we sort the image sequence w.r.t. feature values. We define the error for the image sequence to be $1 / 2$ of the absolute difference to avoid double counts in the ordinal position as assigned by the feature versus that of the ordinal position. The sum of the per-image deviations over the sequence provides the error score for the feature for that image sequence. We perform the same calculations for 19 construction zones separately and obtain the overall error. We obtain the total correct sort value by subtracting the overall error from the total number of images (69 here). Finally, we obtain the percentage of the total correct sort to the total number of images. We indicate it as the average performance of the feature.

We obtain the following overall performance values for $m_{1}$ to $m_{5}$ as $75.4 \%, 76.8 \%, 76.8 \%, 78.3 \%$, and $78.3 \%$, respectively. Although
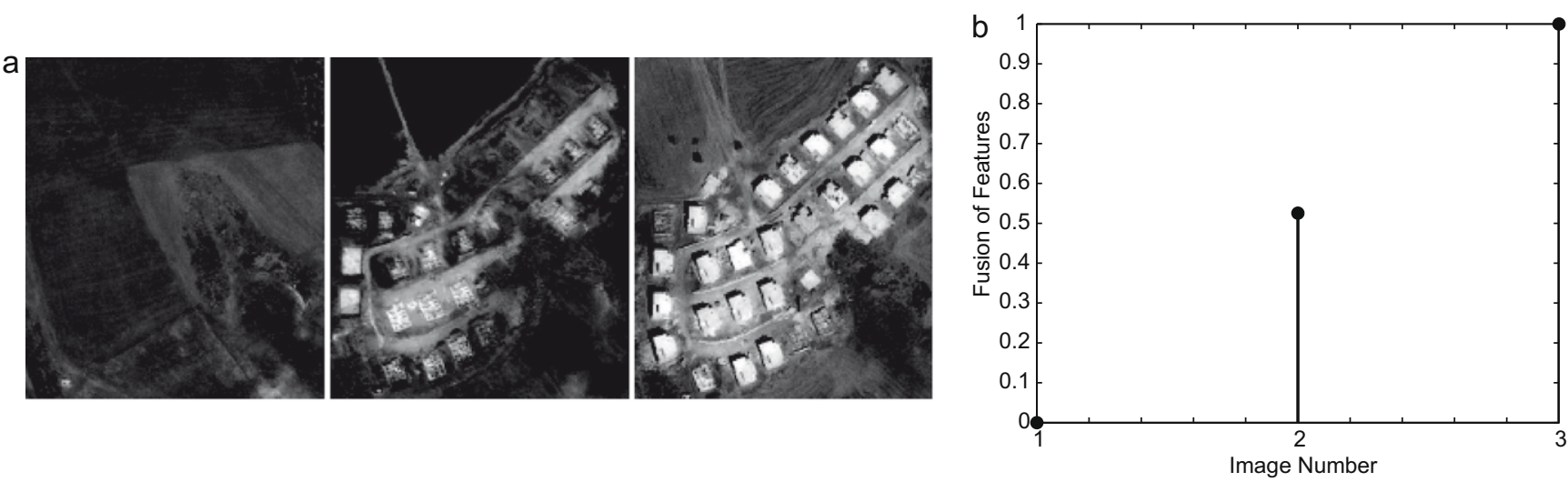

C

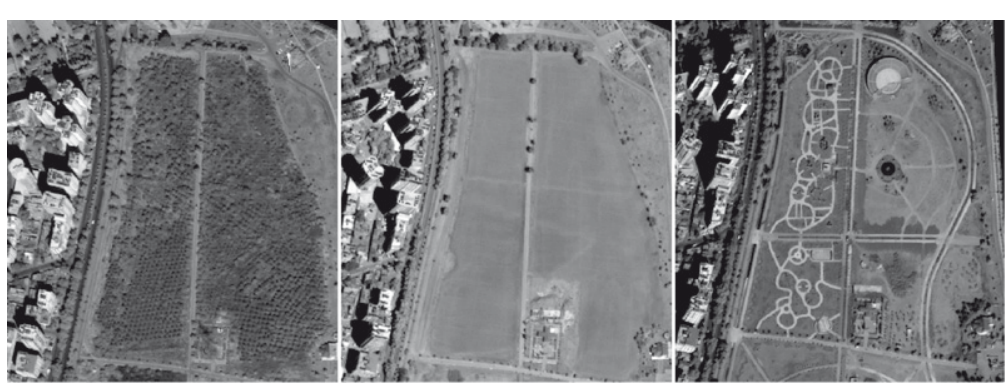

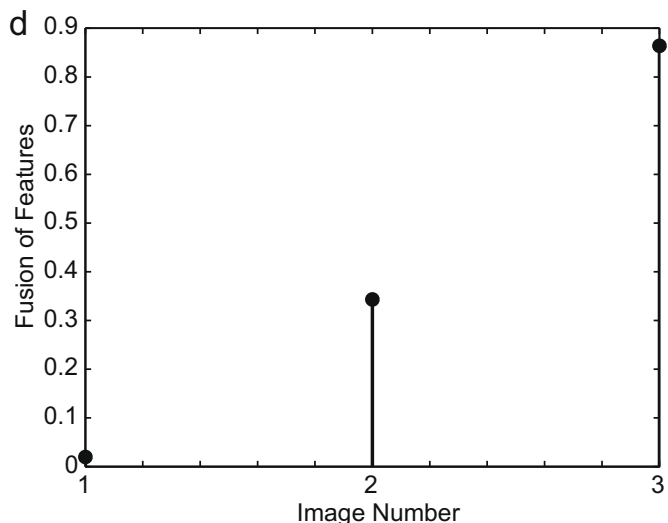

Fig. 3. Test sequences of $A d a n a_{1}$ (first row) and $A_{d a n a}$ (second row) and fusion of land development measures calculated on them. 
the performance values are close to each other, the normalized urban area measure, $m_{4}$, and the normalized sum of votes in the urban region measure, $m_{5}$, have slightly better performance values. As we fuse all the measures, we obtain an $82.3 \%$ performance. Therefore, we gain a $4 \%$ performance improvement after fusion.

\subsection{Comparison with the previous methods}

In our previous graph theoretical land development measures, we obtained the best performance as $72.5 \%$ using one measure alone (Ünsalan, 2007). Our land development measures introduced in this study have at least $2.9-5.8 \%$ better performances compared to the graph theoretical ones. Similarly, with our hybrid multispectral land development measures we obtain a $79.1 \%$ performance using one measure alone (Ünsalan, 2009). Again, performance values obtained in this study are comparable with this result. We only have a $0.8 \%$ decrease in performance. However, we do not benefit from the multispectral information in this study.

In terms of the computational cost measured in CPU times, we reported that graph theoretical land development measures need $59.561 \mathrm{~s}$ on average (over 69 images). Hybrid land development measures need $13.906 \mathrm{~s}$ on average with the same data set and the same computer (a PC with Intel Core2Due Processor with $2.2 \mathrm{GHz}$ clock speed). Our local feature based land development measures need $2.168 \mathrm{~s}$ on average with the same settings. This test indicates that, the computational cost of our new land development measures are also remarkably low compared to previous methods.

\section{Conclusions}

In this study, we present a novel method to measure land development in urban regions. In order to measure development, we use very high resolution panchromatic satellite image sequences of the region. Our method depends on local feature extraction using Gabor filtering. We use these local features in forming a spatial voting matrix. Then, we define five different land development measures on it. After extensive testings, we observe that our novel land development measures have similar or better performances compared to previous land development measures in the literature. Besides, the computational cost of our novel land development measures is fairly low compared to previous methods. Also, we do not benefit from multispectral information as in previous studies. Therefore, land development measures based on local features introduced in this study have their strengths compared to previous ones. Unfortunately, for image sequences with extreme illumination changes, the present method can not perform as good as the multispectral information based method. This constraint should be taken into account in applications. The next step in this study will be detecting specific changes in satellite image sequences.

\section{References}

Kosugi, Y., Sakamoto, M., Fukunishi, M., Lu, W., Doihara, T., Kakumoto, S., 2004. Urban change detection related to earthquakes using an adaptive nonlinear mapping of high resolution images. IEEE Geosci. Remote Sensing Lett. 1, 152156.

Kyrki, V., Kamarainen, J.K., Kalviainen, H., 2004. Simple Gabor feature space for invariant object recognition. Pattern Recogn. Lett. 25 (3), 311-318.

Li, W., Li, X., Wu, Y., Hu, Z., 2006. A novel framework for urban change detection using VHR satellite images. Proc. ICPR'06 2, 312-315.

Mura, M.D., Benediktsson, J.A., Bovolo, F., Bruzzone, L., 2008. An unsupervised technique based on morphological filters for change detection in very high resolution images. IEEE Geosci. Remote Sensing Lett. 5, 433-437.

Otsu, N., 1979. A threshold selection method from gray-level histograms. IEEE Trans. System Man Cyber. 9 (1), 62-66.

Radke, R.J., Andra, S., Al-Kofahi, O., Roysam, B., 2005. Image change detection algorithms: A systematic survey. IEEE Trans. Image Process. 14 (3), 294-307.

Sırmaçek, B., Ünsalan, C., 2009. Urban area and building detection using SIFT keypoints and graph theory. IEEE Trans. Geosci. Remote Sensing 47 (4), 11561167.

Sonka, M., Hlavac, V., Boyle, R., 2007. Image Processing, Analysis and Machine Vision, third ed., CL Engineering.

Tang, F., Prinet, V., 2007. Computing invariants for structural change detection in urban areas. Urban Remote Sensing Joint Event, 1-6.

Ünsalan, C., 2007. Measuring land development in urban regions using graph theoretical and conditional statistical features. IEEE Trans. Geosci. Remote Sensing 45 (12), 3989-3999.

Ünsalan, C., 2009. Statistical, structural, hybrid, and graph theoretical features to measure land development. IEEE Geosci. Remote Sensing Lett. 6 (1), 72-76.

Vetterli, M., Kovacevic, J., 1995. Wavelets and Subband Coding, first ed., Prentice Hall. 